\title{
Strain and thermal conductivity in ultrathin suspended silicon nanowires
}

\author{
Daniel Fan, ${ }^{1,2,{ }^{*}}$ Hans Sigg, ${ }^{1}$ Ralph Spolenak, ${ }^{2}$ and Yasin Ekinci ${ }^{1}$ \\ ${ }^{1}$ Laboratory for Micro and Nanotechnology, Paul Scherrer Institut, Villigen-PSI 5232, Switzerland \\ ${ }^{2}$ Laboratory for Nanometallurgy, ETH Zurich, Zurich 8052, Switzerland \\ (Received 6 June 2017; revised manuscript received 27 July 2017; published 15 September 2017)
}

\begin{abstract}
We report on the uniaxial strain and thermal conductivity of well-ordered, suspended silicon nanowire arrays between 10 to $20 \mathrm{~nm}$ width and $22 \mathrm{~nm}$ half-pitch, fabricated by extreme-ultraviolet (UV) interference lithography. Laser-power-dependent Raman spectroscopy showed that nanowires connected monolithically to the bulk had a consistent strain of $\sim 0.1 \%$, whereas nanowires clamped by metal exhibited variability and high strain of up to $2.3 \%$, having implications in strain engineering of nanowires. The thermal conductivity at room temperature was measured to be $\sim 1 \mathrm{~W} / \mathrm{m} \mathrm{K}$ for smooth nanowires and $\sim 0.1 \mathrm{~W} / \mathrm{m} \mathrm{K}$ for rougher ones, similar to results by other investigators. We found no modification of the bulk properties in terms of intrinsic scattering, and therefore, the decrease in thermal conductivity is mainly due to boundary scattering. Different types of surface roughness, such as constrictions and line-edge roughness, may play roles in the scattering of phonons of different wavelengths. Such low thermal conductivities would allow for very efficient thermal energy harvesting, approaching and passing values achieved by state-of-the-art thermoelectric materials.
\end{abstract}

DOI: 10.1103/PhysRevB.96.115307

\section{INTRODUCTION}

Silicon nanowires (SiNWs) are a versatile functional material, widely studied and employed as, for example, solar cells [1-5], battery anodes [6,7], microelectromechanical systems (MEMS) [8], chemical and biosensors [9-14], thermoelectric harvesters [15-17], and transistors [18-20], due to changes in materials properties in the micro- and nanostructured size regime [21-26]. For transistors, SiNWs provide opportunity for gate-all-around structures, which improve the electrostatic control of the conduction channel, thereby enhancing performance and lowering power consumption. Combining SiNWs with strain allows not only carrier mobility enhancement but also the possibility of optical lasing in a material which has a nondirect band gap in bulk [27]. Silicon nanowires also have excellent thermoelectric properties, exhibiting a low thermal conductivity due to increased phonon scattering, high electrical conductivity, and combined with a high Seebeck coefficient allows efficient thermal energy harvesting [16,28,29]. Thermoelectric figures of merit up to $Z T \approx 0.6$ at room temperature have been reported, compared to the bulk silicon value of $Z T \approx 0.01$ [30]. One method of nondestructive materials characterization is Raman spectroscopy. Extensive Raman work has been done on SiNWs, where the Raman peak shift and shape can be related to strain, thermal effects, and confinement effects [17,29-50]. The largest and most common contributor to Raman peak shift and broadening are thermal effects such as those due to local laser heating or insufficient thermal contact between SiNW and substrate [40]. For SiNWs below $15 \mathrm{~nm}$, quantum confinement begins to have an observable effect, causing peak shifts and asymmetric peak broadening [51-53]. It is also known that strain, depending on the crystal direction, can be related to peak shifts for the different optical mode phonons [54-56]. Much of the work done using Raman on SiNWs has been on chemically synthesized or chemically etched SiNWs, which typically

\footnotetext{
*daniel.fan@psi.ch
}

exhibit a size, position, and orientation distribution. Therefore, the Raman spectra require further deconvolution to account for these geometric distributions within the laser probe spot [57].

The processing infrastructure for silicon is well established. However, SiNWs below $20 \mathrm{~nm}$ width, which have been studied in the recent past, have been typically fabricated using electrochemical etching $[30,31,44-47,49,50]$ or bottomup synthesis $[1,22,23,32,33,37,39,42-44,48]$, making them difficult to incorporate into devices, having a larger distribution of sizes and orientations, lacking control over placement, and restricting the crystal orientations and doping levels available. For top-down methods, the feature size has been limited by the optical lithography wavelength which is currently deep-ultraviolet (UV; $\lambda=193 \mathrm{~nm}$ ). Great efforts have been made by the industry to move to the extreme-UV (EUV) wavelength $(\lambda=13.5 \mathrm{~nm})$ for resolution improvement [58,59]. The highest resolution features can be obtained using interference lithography (IL), where two coherent beams interfere with each other and the interference pattern is recorded. Combined with EUV, this allows the ability to print well-ordered and highly dense periodic patterns over a large area [60,61]. Measurement techniques can therefore achieve very high signal-to-noise ratios as compared to single SiNWs, while the strict order allows studies of specific sizes and orientations. Line/space patterns down to $6 \mathrm{~nm}$ half-pitch have been demonstrated using this method [62], allowing accurate placement of nanostructures with precise dimensions.

\section{MATERIALS AND METHODS}

Large area, highly dense line/space patterns at $22 \mathrm{~nm}$ half-pitch were printed using EUV-IL at the XIL-II beamline, Swiss Light Source, Paul Scherrer Institut, for which the scheme is described elsewhere [60]. Briefly, coherent EUV light is diffracted by two gratings, the two diffracted beams then interfere, and the interference pattern is recorded in photoresist [Fig. 1(a)]. Hydrogen silsesquioxane negative tone photoresist (Dow Corning, Midland, U.S.A.) diluted 1:1 with methyl isobutyl ketone (Dow Corning) was spin-coated onto 


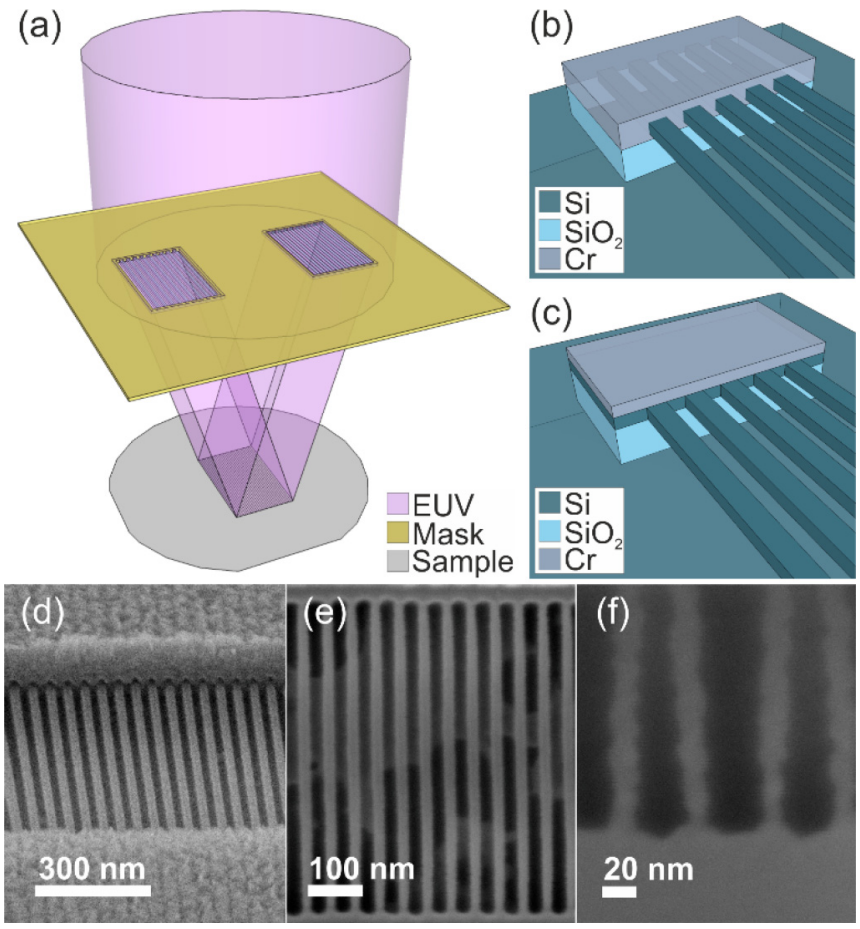

FIG. 1. (a) EUV-IL scheme, not to scale. Coherent EUV light is incident upon two gratings which diffract the beams such that they interfere. The interference pattern is then recorded. (b) Schematic of SiNWs clamped by $\mathrm{Cr}$ and released by VHF or BOE etching. (c) Schematic of SiNWs connected monolithically to Si pads. (d) SEM of SiNWs clamped by $\mathrm{Cr}$ where an air gap is apparent between $\mathrm{SiNW}$ and metal due to underetching. (e) SEM of SiNWs connected to Si pads and released by VHF, showing smooth surfaces but constrictions at both ends. (f) SEM of SiNWs connected to Si pads and released by BOE followed by width thinning in Piranha solution and then critical point drying. The resulting LER value is relatively high i.e. $\sim 4 \mathrm{~nm}$.

a silicon-on-insulator substrate $[25 \mathrm{~nm}$ device layer, $145 \mathrm{~nm}$ buried oxide, $725 \mu \mathrm{m}$ handle silicon, (100), lightly $p$ doped to $10^{15} \mathrm{~cm}^{-3}$, SOITEC, France] for a target thickness of $20 \mathrm{~nm}$. The line/space pattern was exposed via EUV-IL and developed using Microposit 351 developer (Dow Corning) diluted 1:3 with deionized water. The pattern was transferred into the device layer via inductively coupled plasma reactive ion etching (ICP-RIE, Oxford100, $20 \mathrm{sccm} \mathrm{SF}_{6}, 50 \mathrm{sccm} \mathrm{C}_{4} \mathrm{~F}_{8}$, $0{ }^{\circ} \mathrm{C}, 18 \mathrm{mTorr}, 600 \mathrm{~W}$ ICP power, $25 \mathrm{~W}$ RF power, Oxford Instruments, U.K.). Chromium anchor pads of $50 \mathrm{~nm}$ thickness were defined by e-beam lithography (EBPG 5000+, Vistec, Jena, Germany), deposited by thermal evaporation (Balzers, Oerlikon, Switzerland), and the unwanted areas lifted off in acetone (Technic France, France). The nanowires were then release by etching the buried oxide either via wet etching using buffered oxide etch (BOE; HF1 : 7 $\mathrm{NH}_{4} \mathrm{~F}, 2$ min, Technic France) or vapor hydrofluoric acid (VHF) etch (5 min, Technic France). This gave suspended SiNWs that were clamped down by $\mathrm{Cr}$ pads [Fig. 1(b)]. Another approach for anchoring was used by first defining the anchor areas using e-beam lithography, metal deposition combined with lift-off, followed by ICP-RIE pattern transfer and release in HF. This gave suspended SiNWs that were monolithically connected to the bulk silicon with $\mathrm{Cr}$ on top of the silicon pads [Fig. 1(c)]. The
$\mathrm{Cr}$ metal was required to improve discharging during scanning electron microscope (SEM) imaging. Scanning electron microscopy was performed at $1 \mathrm{kV}$ acceleration voltage and $3.5 \mathrm{~mm}$ working distance (Supra V55, Zeiss, Oberkochen, Germany).

The fabricated specimens were measured using a homemade micro-Raman spectroscopy setup based on a $405 \mathrm{~nm}$ single-mode continuous wave laser (Integrated Optics, Vilnius, Lithuania). An inverted microscope (Leica DMI5000 M, Wetzlar, Germany) with $100 \times$ objective, numerical aperture $(\mathrm{NA})=0.9$, was used as the sample stage, followed by a $405 \mathrm{~nm}$ notch filter (Edmund Optics, York, U.K.) to allow both Stokes and anti-Stokes detection, then a $40 \mu \mathrm{m}$ slit entrance onto an 1800 lines/mm spectrograph (Acton SP500i, Princeton Instruments, U.S.A.). The light was recorded by a singlephoton charge coupled device (CCD) camera (ProEM 1600², Princeton Instruments) and controlled using WinSpec software (Princeton Instruments). Summation of three $30 \mathrm{~s}$ exposures was sufficient to achieve acceptable signal-to-noise ratio, and the laser power was measured at the sample using a power meter (Fieldmaster, Coherent, Santa Clara, U.S.A.). Two half-wave plates at the entrance and exit of the microscope allowed polarization dependent measurements. The SiNWs were imaged by SEM and analyzed using a commercial SEM image analysis software (SuMMIT, EUV Tech, Martinez, U.S.A.) to obtain the SiNW width and the line-edge roughness (LER). Due to the dielectric mismatch between SiNW and air, a large polarization anisotropy is exhibited, and the laser polarization was aligned along the direction of the SiNW for maximum signal intensity (see Supplemental Material [63]). Power absorption in the SiNWs was calculated using rigorous coupled-wave analysis (see Supplemental Material [63] and Refs. [64-67]). The spot size was measured to be $1.78 \mu \mathrm{m}^{2}$ with a power range between 0 and $100 \mu \mathrm{W}$ falling on the sample.

\section{EXPERIMENTAL}

In this paper, large area suspended SiNW arrays at $22 \mathrm{~nm}$ half-pitch and widths between 8 and $24 \mathrm{~nm}$ were fabricated by EUV-IL [Fig. 1(a)], etched using RIE into a lightly $p$-doped, (100), $20 \mathrm{~nm}$ thick silicon-on-insulator substrate, and studied by Raman spectroscopy (further details in Materials and Methods). The properties of SiNWs resulting from different methods of SiNW anchoring and underetch release were evaluated, and substantial impact of the processing method on material properties was observed. The $\mathrm{SiNW}$ arrays were either clamped by metal deposition [Fig. 1(b)] or monolithically anchored to bulk silicon pads at either end [Fig. 1(c)], followed by structure release by etching the buried oxide using VHF etch or wet BOE.

Scanning electron microscopy (SEM) images of the typical structures are shown in Figs. 1(e)-1(f). Silicon nanowire arrays clamped by $\mathrm{Cr}$ metal and released by $\mathrm{BOE}$ are shown in Fig. 1(d), SiNW arrays monolithically connected to bulk Si pads and released by VHF in Fig. 1(e), and SiNW arrays monolithically connected to bulk $\mathrm{Si}$ pads and undergoing Piranha oxidation and buffered oxide etching for decreasing the width followed by critical point drying shown in Fig. 1(f). A summary of the sample fabrication variations and their 
TABLE I. Fabrication variations and the resulting properties for suspended SiNWs.

\begin{tabular}{lll}
\hline Anchoring methods & Release methods
\end{tabular}

Monolithic Si pads: SiNWs monolithically connected to large silicon pads at both ends. On top of the silicon pads is $75 \mathrm{~nm}$ of chromium for discharging during SEM inspection. Constrictions can appear at the ends due to etching process.

Clamping Cr pads: SiNWs are etched first and then clamped by metal deposition at both ends. There are no constrictions at the ends. However, since the SiNWs in the anchoring area have an interface between the metal above and the oxide below, an air gap may form during release etching.

properties are described in Table I. The SEM images were used for quantitative evaluation of LER and nanowire width.

The Raman spectra were recorded (Fig. 2) for various laser powers, the Stokes/anti-Stokes peak intensities $\left(I_{S}, I_{A S}\right)$ were measured, and the temperature was calculated using the equation [44]

$$
\frac{I_{\mathrm{S}}}{I_{\mathrm{AS}}}=C\left(\frac{\omega_{\mathrm{S}}}{\omega_{\mathrm{AS}}}\right)^{3} \exp \left(\frac{\hbar c \omega}{k_{B} T}\right)
$$

where $\omega_{\mathrm{S}}$ and $\omega_{\mathrm{AS}}$ are the Stokes and anti-Stokes photon frequencies, respectively, $\omega$ is the phonon frequency, $C$ is a
VHF: uses HF in the vapor phase to etch the buried oxide without causing stiction. The resulting LER is typically low.

BOE: $\left(\mathrm{HF} 1: 7 \mathrm{NH}_{4} \mathrm{~F}\right)$ in liquid state to etch the buried oxide. The specimen is placed in isopropanol last for quick drying. The yield is low due to stiction from capillary forces during drying. The LER is also low.

CPD: involves Piranha oxidation for width reduction and BOE, while the last step is critical point drying to avoid stiction. The LER is high.

constant determined by the absorption constants and Raman cross-sections, and $k_{B}, \hbar$, and $c$ are the constants of Boltzmann, Planck, and the speed of light, respectively. The calculated temperature was plotted as solid data points in Fig. 2 and linearly fitted by solid lines. The Raman spectra were obtained at small laser power, increasing the laser power, then going back to small laser power to observe any hysteresis or phase change in the material. It was observed that, at $\sim 450 \mathrm{~K}$, the Raman spectrum changed, and the original low power spectra could not be reproduced, indicating a phase change or breakage in the material (see Supplemental Material [63]). These data points were therefore ignored in the linear fit. The linear fit
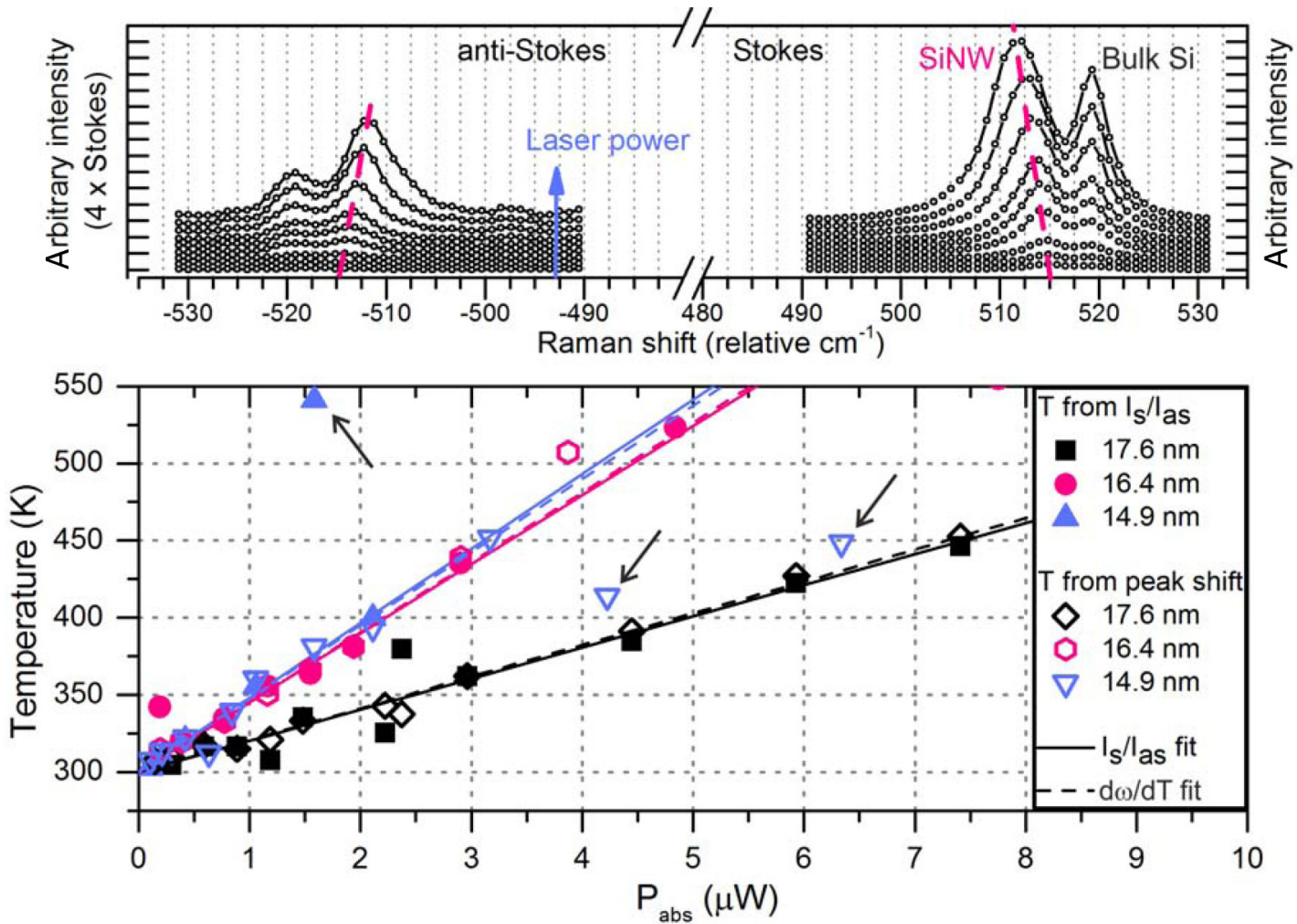

FIG. 2. Top: example laser-power-dependent Raman spectra of suspended SiNWs with the dotted lines highlighting the SiNW Raman peak shift as laser power is varied. Bottom: measurement of temperature using Stokes/anti-Stokes peak intensities (solid) and Raman peak shift (hollow, dotted). The data points highlighted by arrows were ignored in the fitting due to hysteresis—as the laser power was cycled high and then low, the original low power spectra could not be recovered, possibly due to materials changes caused by high temperatures. 
was adjusted for parameter $C$ in Eq. (1) so that the zero-power intercept was at room temperature.

Similarly, the Stokes/anti-Stokes peak positions for each laser power was related to a temperature using the bulk silicon relation of $\frac{d \omega}{d T}=-0.024 \mathrm{~cm}^{-1} / \mathrm{K}[68,69]$, independent of intensity, where the temperature-dependent part of the Raman peak shift at room temperature can be approximated by the linear relation [44]

$$
\frac{d \omega}{d T} \approx-3 \omega_{0} \gamma \alpha+\frac{k_{B}}{\hbar c \omega_{0}}[4 A+9 B],
$$

where $T$ is the temperature, $\alpha$ is the coefficient of linear thermal expansion, $\omega_{0}$ is the zone center phonon frequency, $\gamma$ is the Grüneisen parameter, and $A$ and $B$ are the cubic and quartic harmonic constants, respectively. The temperature calculated using the Stokes peak shift are plotted as hollow data points in Fig. 2 and linearly fitted by dotted lines. These two independent measurements of temperature agree well, similar to the work by Doerk et al. [44], who found the same bulk silicon relation of Raman peak shift to temperature for electrochemically etched SiNWs down to $50 \mathrm{~nm}$ diameter. Doerk et al. suggested that, as per Eq. (2) above and considering the matching measurements of temperature, the anharmonic constants for SiNWs remain bulklike, an indication that lattice anharmonicity is not modified compared with bulk values. Similarly, from the first term of Eq. (2), the Grüneisen parameter is bulklike, and the bulk silicon value can be taken, i.e. $\gamma \approx 0.98[68,70]$. Thus, $\frac{d \omega}{d T}=0.024 \mathrm{~cm}^{-1} / \mathrm{K}$ can be taken for subsequent measurements of temperature where the anti-Stokes intensity is insufficient for accurate calculation of temperature.

The laser-power-dependent Raman peak shift can be fitted linearly, shown in Fig. 3 for a few SiNW examples. The thermal conductivity is thus approximately constant in the temperature ranges being investigated, and Eq. (2) is valid. At higher temperatures $>400 \mathrm{~K}$, the peak shift could be better fitted quadratically, indicating changes in the thermal conductivity due to increased phonon-phonon scattering at higher temperatures. These data points were ignored in the linear fit. The slope can be related to thermal conductivity, while the zero-power intercept represents the Raman peak shift in the absence of any thermal effects and is thus composed of quantum confinement effects and uniaxial elastic strain [71]. Combining a phonon confinement model based on bond polarizability [53] and a simple uniaxial strain model [38], the Raman shift due to quantum confinement and uniaxial elastic strain for a $\mathrm{SiNW}$ is

$$
\Delta \omega=-A\left(\frac{a_{0}}{D_{w}}\right)^{\beta}-n \gamma \omega_{0} \frac{a-a_{0}}{a_{0}},
$$

where $\Delta \omega$ is the phonon shift from the optical phonon frequency at the zone center, $\omega_{0}$ is the optical phonon frequency at the zone center, $a_{0}$ is the bulk silicon lattice constant, $a$ is the SiNW lattice constant, $D_{w}$ is the SiNW diameter for a circular $\mathrm{SiNW}, n$ is the dimensionality (for SiNWs $n=1), \gamma$ is the Grüneisen parameter, and $A=20.92$ and $\beta=1.08$ are fitting parameters. Here, $D_{w}$ can be replaced for a SiNW with rectangular cross-section (width $w$, height $t$ )
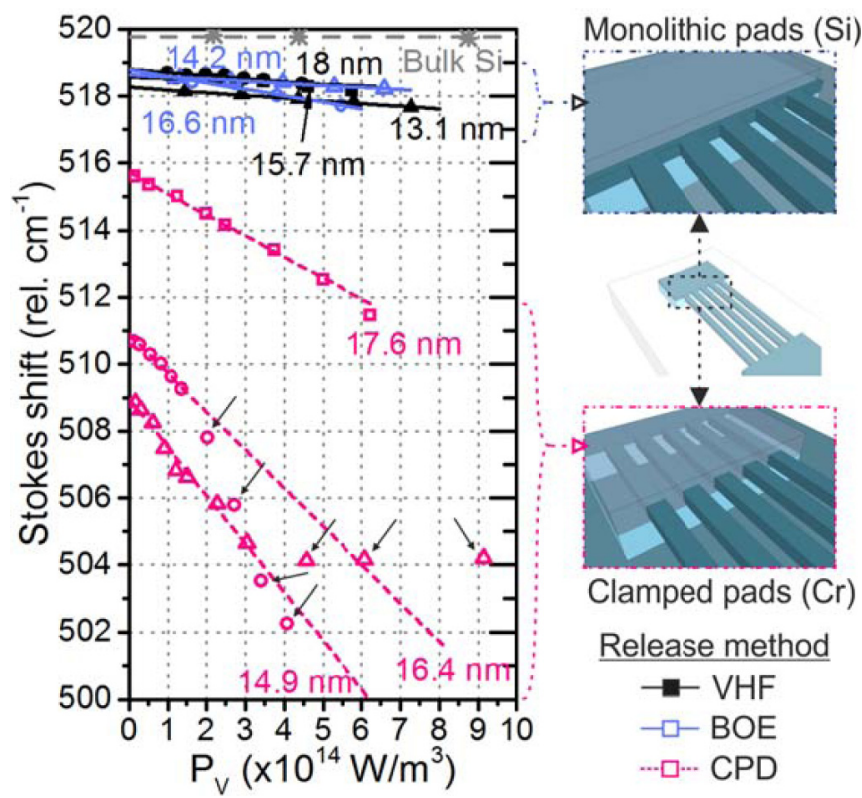

FIG. 3. Example laser-power-dependent Stokes peak shift for a variety of SiNWs processed using different methods. The SiNWs are either joined monolithically to bulk silicon pads or clamped by $\mathrm{Cr}$ metal and released by either VHF, wet BOE, or wet BOE combined with critical point drying. The data points highlighted by arrows were ignored in the fit due to hysteresis of the Raman spectra, indicating materials phase changes possibly due to the high temperatures.

with the effective size

$$
D_{w}=\frac{2 \sqrt{w t}}{\sqrt{\pi}},
$$

The bulk silicon Raman shift for the anchoring pads and substrate was measured to be $519.75 \mathrm{~cm}^{-1}$. Taking this as the zone center phonon frequency $\omega_{0}$, the quantum confinement was calculated using the first term of Eq. (3) and plotted against width in Fig. 4 (dotted line). Any subsequent zero-laser-power Raman peak shift is therefore due to uniaxial elastic strain, described by the second term in Eq. (3). Raman back-scattering from a (001) silicon surface allows only the longitudinal optical (LO) mode to be observed [56], while using a high NA objective with small spot size allows measurement of the two transverse optical (TO) modes as well [54]. Although Tarun et al. showed that prestrained SiNWs retain a complex strain distribution when patterned on oxide [54], only uniaxial strain is expected to remain after suspension of the SiNWs, whereby the free surfaces of the SiNW are allowed to relax. Gridlines for uniaxial elastic strain in intervals of $0.5 \%$ are plotted in Fig. 4 in blue.

\section{A. Strain}

The zero-laser-power Raman peak shift for various SiNW arrays is plotted against SiNW width (Fig. 4). Clearly, the difference in SiNW anchoring plays an important role. For SiNWs anchored and joined monolithically to the bulk silicon (solid data points, Fig. 4), the Raman shift is small and close to the bulk silicon value for all SiNW widths in the range from 10 to $20 \mathrm{~nm}$. A slight downward trend as the width 


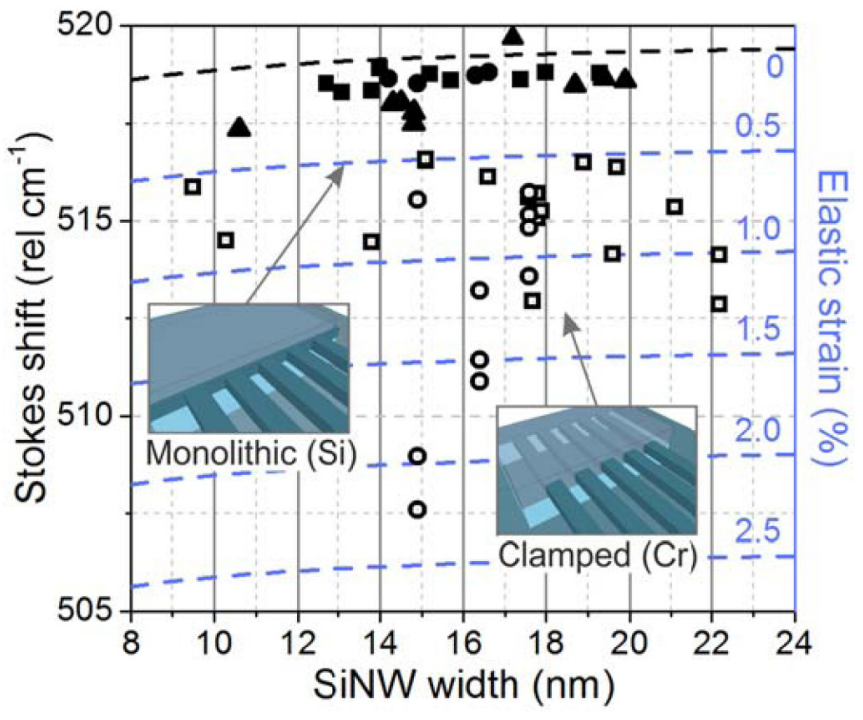

\begin{tabular}{|ll|cc|}
\hline - & Si pads, VHF & a & Cr pads, VHF \\
- & Si pads, BOE & ○ & Cr pads, BOE \\
- & Si pads, CPD & - & - Q.C. model \\
\hline
\end{tabular}

FIG. 4. SiNW width vs extrapolated zero-laser-power Stokes shift. Estimate of the quantum confinement component (thick dotted line) and the uniaxial elastic strain (blue dotted lines) are plotted. Monolithically connected SiNWs (solid) have low strain. Metal clamped SiNWs (hollow) exhibit high and variable process-induced strain. Very rough SiNWs (solid triangle) have low but variable strain, possibly due to variable oxide formation.

decreases correlates well with quantum confinement effects. A consistent elastic strain of approximately $0.1 \%$ is apparent. For silicon-on-insulator (SOI) substrates, the silicon device layer might be slightly prestrained due to the processing method. The release of the biaxial strain in one in-plane direction can affect the uniaxial strain in the orthogonal direction, a technique used on heavily prestrained substrates to fabricate strained nanowires [72]. It has also been reported that oxidizing the nanowire shell can impart stress along the nanowire [35,49]. For the SiNWs released by VHF and BOE, the scatter in strain is fairly small, since the size of the native oxide shell is consistent. For SiNWs undergoing critical point drying (solid triangles, Fig. 4), the surface roughness is large [Fig. 1(f)], causing different thicknesses of oxide shell along the nanowire, which may impart different types of stresses, thereby causing inconsistent strain.

For SiNWs clamped by thermally evaporated metal (hollow data points, Fig. 4), the Raman shift and hence strain is significant, in some cases up to $2.3 \%$ uniaxial elastic strain. The variability in the strain is large, and for SiNWs released by BOE underetching (hollow circles, Fig. 4), the strain varies significantly over a small SiNW width range. One possible explanation for the increased strain in SiNWs which are clamped is thermal expansion of the SiNW structure during thermal evaporation of the metal, which then clamps the SiNW and thus keeps it strained after the sample has cooled, or thermal contraction of the clamping metal. Although variability is an issue, this has important consequences for strain engineering of materials, for example, for carrier mobility enhancement in transistor devices [73] and enhanced optical properties [27]. Direct metal deposition represents another method for electrical contacting of ultrathin SiNWs. By using an unstrained substrate and etching nanometer-sized features and applying a thermal processing budget to thermally expand the material, one can achieve significant strain. Currently, strain engineering typically involves using prestrained substrates which are epitaxially grown or wafer-bonded and which can be expensive [74].

\section{B. Thermal conductivity}

The suspended SiNWs had a maximum length of $600 \mathrm{~nm}$, smaller than the laser spot size of $1.78 \mu \mathrm{m}$. Therefore, the SiNWs are assumed to be homogeneously heated by the laser. The thermal contact resistance was also modeled using finite element methods, showing that SiNWs monolithically connected to bulk Si pads have their boundary conditions essentially pinned to room temperature (see Supplemental Material [63]). The heat equation with room temperature boundary conditions is then applied to find the average temperature across the nanowire array, and subsequently an expression for the thermal conductivity [75]

$$
\kappa=\frac{P_{V} L^{2}}{12\left(T_{\text {avg }}-T_{0}\right)},
$$

where $\kappa$ is the thermal conductivity, $P_{\mathrm{V}}$ is the absorbed power per unit volume in the SiNW, $T_{\text {avg }}$ is the average temperature over the whole SiNW, $L$ is the length of the SiNW, and $T_{0}$ is the SiNW boundary temperature which was calculated to be fixed. The incident laser power was measured using a power meter and the spot size measured using the knife edge method. The absorbed power was calculated using rigorous coupled wave analysis $[64,76]$ for the suspended SiNW array. A series of measurements of the temperature of the system for each absorbed power was linearly fitted to give the thermal conductivity at room temperature.

For bulk silicon, the measured change in temperature was small, and the thermal conductivity was measured to be $\sim 150 \mathrm{~W} / \mathrm{m} \mathrm{K}$, as expected following standard measurements in literature. For SiNWs, the thermal conductivity was measured to be in the range of 0.04 to $1.5 \mathrm{~W} / \mathrm{m} \mathrm{K}$, depending on the LER, which is $2-3$ orders of magnitude lower than the bulk value. For SiNWs connected to bulk silicon anchor pads and released using various methods, $\kappa$ is plotted against the width in Fig. 5. The LER was measured using SEM analysis software to be $\sim 2 \mathrm{~nm}$ for SiNWs released by VHF and BOE, and between 3.4 to $4.6 \mathrm{~nm}$ for SiNWs undergoing Piranha thinning and critical point drying. There does not appear to be strong SiNW width dependence in this width range. For SiNWs released using VHF, giving smooth sidewalls, the thermal conductivity is approximately $1 \pm 0.5 \mathrm{~W} / \mathrm{m} \mathrm{K}$. For the rougher SiNWs, $\kappa$ is approximately $0.1 \pm 0.05 \mathrm{~W} / \mathrm{m} \mathrm{K}$. For SiNWs clamped by $\mathrm{Cr}$, the thermal conductivity could not be calculated because an air gap was formed between the SiNW and the metal pad [Fig. 1(d)], resulting in an unknown boundary condition (see Supplemental Material [63]). Overall, the decrease in $\kappa$ for the SiNW array agrees well with the trend of decreasing $\kappa$ vs width reported by a variety of studies of SiNWs synthesized by various methods [Fig. 4(b)] 


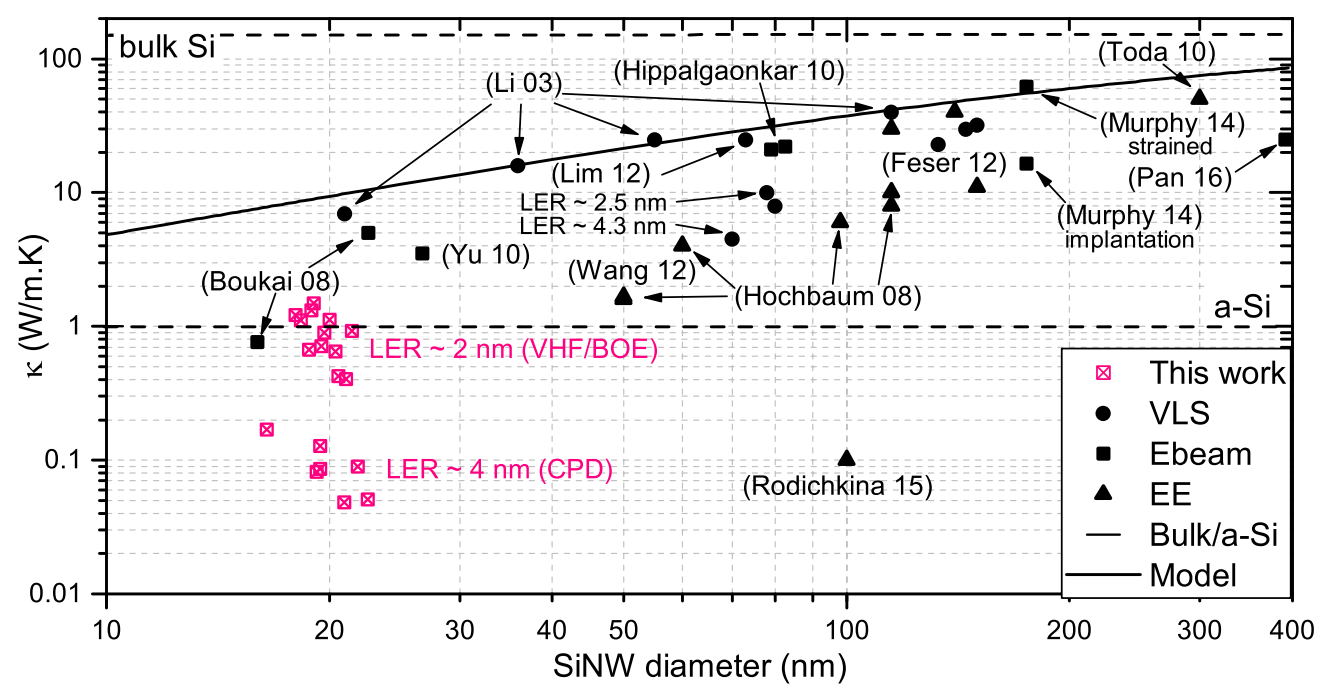

FIG. 5. Thermal conductivity vs width for SiNWs connected monolithically to Si pads including data from various references. A model of the thermal conductivity due to purely diffusive scattering (Casimir limit) is shown (solid line). The SiNWs released by VHF and BOE had a LER of $\sim 2 \mathrm{~nm}$, while SiNWs undergoing critical point drying had a LER of $\sim 4 \mathrm{~nm}$. For e-beam defined SiNWs with rectangular cross-section, the effective diameter was calculated using Eq. (4) for better comparison.

[29-31,36,48-50,77-81]. The degree of scatter in the data is approximately $\pm 50 \%$, slightly larger than simulations which show sensitivity errors of $\sim \pm 30 \%$.

For insulators and semiconductors, heat transport is mediated mainly by phonons. Using Matthiessen's rule, the total phonon scattering rate is the sum of intrinsic, boundary, crystal defects (such as grain boundaries), phonon-electron, and impurity scattering rates, and the quantum confinement effect where the phonon dispersion itself is modified. Since the nanowires are above $10 \mathrm{~nm}$ in size, confinement effects are negligible. Since the SiNWs are etched from bulk crystalline silicon, grain boundary scattering is expected to be minimal. Since the SiNWs have low doping, the phonon-electron and impurity scattering will also be minimal at room temperature.

Furthermore, it was shown earlier that lattice anharmonicity was not modified and therefore did not play a role in the decrease in thermal conductivity in SiNWs down to $10 \mathrm{~nm}$ widths, supporting the work by Doerk et al. [44]. At such low nanowire dimensions, surface effects are expected to dominate, and boundary-induced changes to lattice anharmonicity would become more apparent. However, no such variations in lattice anharmonicity was observed, and the intrinsic scattering remains bulklike.

Strained suspended SiNWs in the $\langle 110\rangle$ direction were fabricated using a prestrained SOI substrate with the SiNWs monolithically connected to the bulk silicon pads (Fig. 6). The uniaxial elastic strain was measured by Raman to be $\sim 0.75 \%$ compared with the unstrained SiNWs with $\sim 0.15 \%$ strain. The thermal conductivity of the strained SiNWs were measured to be similar in value to the unstrained SiNWs of between $0.3-1 \mathrm{~W} / \mathrm{m} \mathrm{K}$. Similarly, Murphy et al. [36] showed that strain did not modify the thermal conductivity for $\langle 111\rangle$ oriented SiNWs, but bulk ion implantation had a large effect on thermal conductivity. Strain can cause changes to the lattice anharmonicity and affect intrinsic scattering mechanisms. Since no effects due to strain were observed, the intrinsic scattering is bulklike, and therefore, the thermal conductivity for the SiNWs in this paper is mediated mainly by boundary scattering.

From the work by Hochbaum et al. [30], Li et al. [48], and others [17], the temperature dependence of $\kappa$ near room temperature for SiNWs with small widths is relatively constant. In this paper, the measured temperatures of suspended SiNWs with low LER ranged from room temperature up to $\sim 325 \mathrm{~K}$, such that it can be assumed that $\kappa$ is constant in this temperature range. The best fit of Stokes shift (which is proportional to temperature) vs laser power is linear, also indicating that $\kappa$ is constant over this temperature range. For SiNWs with high LER, the temperatures measured ranged up to $\sim 500 \mathrm{~K}$, and the temperature dependence of $\kappa$ must be taken into consideration. Therefore, only measurements at low power were fitted linearly.

\section{Boundary scattering}

As the size of nanowires decreases below the phonon mean free path ( $\sim 300 \mathrm{~nm}$ at room temperature), boundary scattering begins to dominate phonon transport. In the Casimir limit where the boundary scatters completely diffusively, the relative reduction in thermal conductivity due to boundary and intrinsic scattering is [17]

$$
\frac{\kappa_{\text {SiNWs }}}{\kappa_{\text {bulk }}} \approx\left(1+\frac{\Lambda}{D_{w}}\right)^{-1},
$$

where $\Lambda$ is the phonon mean free path and $D_{\mathrm{w}}$ is the nanowire diameter for circular nanowires. For rectangular nanowires with thickness $t$ and width $w$, the effective diameter can be defined by Eq. (2).

The thermal conductivity vs SiNW width using this model is plotted (solid line) in Fig. 5. The experimental values measured on vapor-liquid-solid (VLS) grown circular SiNWs by $\mathrm{Li}$ et al. [48] agree well with the model. Rectangular shaped SiNWs fabricated by lithography definition and RIE as well as electrochemically etched SiNWs, show a $D_{\mathrm{W}}{ }^{2}$ 
(a)

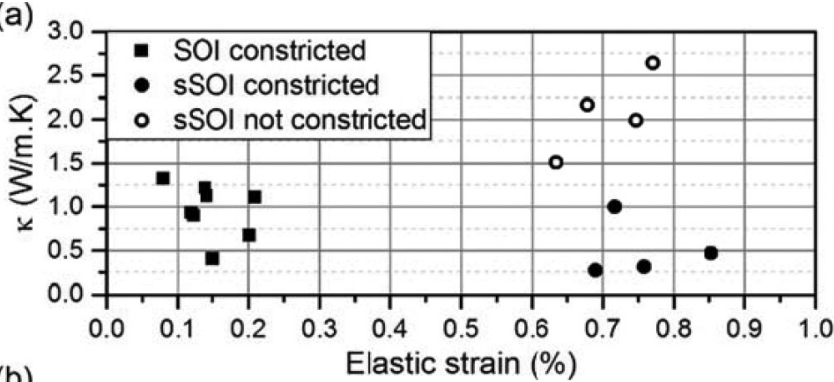

(b)

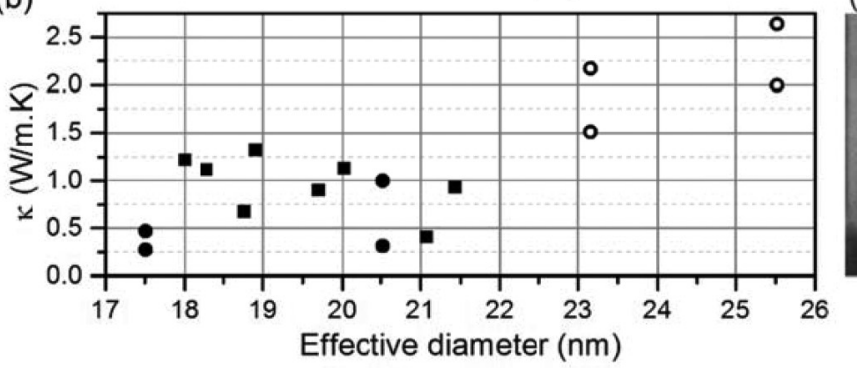

(c)

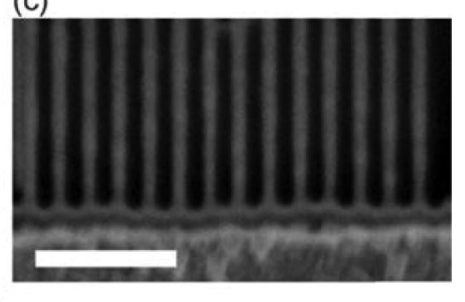

(d)

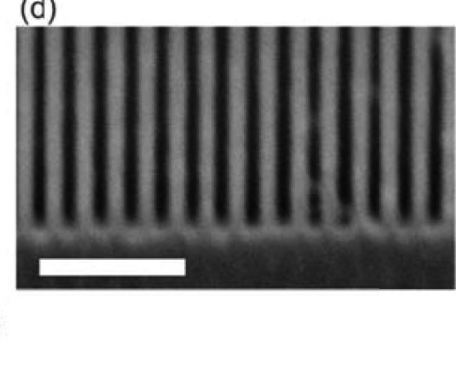

FIG. 6. (a) Uniaxial elastic strain vs thermal conductivity for suspended SiNWs connected monolithically to bulk silicon pads. The strained SiNWs were fabricated from prestrained SOI substrates. Depending on the placement of metal pads for discharging during SEM inspection, the SiNWs exhibited either constrictions at the ends (metal pads, solid) or no constrictions (no metal pads, hollow). (b) Effective diameter [Eq. (4)] vs thermal conductivity for strained and nonstrained SiNWs. (c) Strained $17 \mathrm{~nm}$ width SiNWs without constrictions. (d) Strained $11 \mathrm{~nm}$ width SiNWs with constrictions. Scale bars equal $200 \mathrm{~nm}$. Unstrained substrates indicated by SOI, strained substrates indicated by sSOI.

dependency, i.e. scaling to the nanowire cross-sectional area, corresponding with calculations using perturbation theory by Martin et al. [82]. Electrochemically etched SiNWs are typically doped, and impurity scattering may be a cause of lower thermal conductivity. Lithography defined SiNWs can thus better isolate the effect of boundary scattering. The boundary scattering rate $\tau_{B}{ }^{-1}$ can be expressed as [83]

$$
\tau_{B}^{-1}=\frac{V}{D_{W}} \frac{(1-p)}{(1+p)},
$$

where $V$ is the phonon group velocity, $D_{\mathrm{W}}$ is the SiNW diameter, and $p$ is the surface roughness parameter $(p=1$ for fully specular, $p=0$ for fully diffuse). If the phonon wavelength is much larger than the surface roughness, the phonons are reflected specularly and there is no effect on thermal conductivity. If the phonon wavelength is similar to the surface roughness, the phonons are scattered diffusely, which lowers the probability of phonon transport and hence lowers $\kappa$. If the phonon wavelength is much smaller than the surface roughness, they can be backscattered, giving much lower thermal conductivities than pure diffusive scattering from the surface. For example, SiNWs fabricated by Hochbaum et al. [30], Lim et al. [81], and Feser et al. [79] have a very rough surface and exhibit much lower thermal conductivities than the purely diffuse scattering model. Alternatively, Heron et al. showed that very long wavelength phonons can be backscattered in SiNWs with serpentine structures [80], which can be seen as structures that have a 'roughness' much larger than the phonon wavelength of interest. Therefore, in the case of extremely rough nanowires where the $\mathrm{SiNW}$ is so rough it can be viewed as a row of connected nanocrystals, backscattering becomes important. This increases the scattering rate beyond that predicted by the Casimir limit and could be possibly described phenomenologically by allowing $p<0$ as suggested by Moore et al. [84].

For silicon at room temperature, the phonon wavelength is approximately 1-2 nm, and roughness in the order of 1-2 nm will scatter phonons diffusely. However, the phonon spectrum is also quite broad, and thus phonons of different wavelengths will be scattered by the boundary differently $[81,85,86]$. The data points in Fig. 5 show the thermal conductivity of SiNWs with a relatively smooth surface [Fig. 1(e), LER $\sim 2 \mathrm{~nm}$ ] and rougher surface [Fig. 1(f), LER $\sim 4 \mathrm{~nm}$ ]. Although the LER is much smoother compared to those SiNWs studied by Hochbaum et al. [30], the thermal conductivity is similarly much lower than predicted by the diffuse boundary scattering model. One reason might be because the ends of the nanowires are pinched, causing a slight constriction. This is possibly due to the fabrication process: the SiNWs are patterned, and the metal pads are patterned on top. The whole structure is then etched using plasma etching, where the presence of the metal can modify the local electric field causing overetching at the ends of the SiNWs. These constrictions can act like the serpentine structures studied by Heron et al., i.e. scatter the long-range phonons. In particular, both strained and unstrained SiNWs which exhibited constrictions at the ends had thermal conductivities of approximately $0.5-1 \mathrm{~W} / \mathrm{m} \mathrm{K}$ [Figs. 6(a), 6(c), and 6(d)]. Strained SiNWs that did not exhibit constrictions at the ends, due to processing variations, had larger thermal conductivities (Fig. 6) of around $2 \mathrm{~W} / \mathrm{m} \mathrm{K}$. This shows the role of constrictions in modifying the thermal conductivity.

For SiNWs undergoing critical point drying, the thermal conductivity is much lower due to the much rougher surface, in addition to the constrictions at the ends of the SiNWs. This supports the work by Lim et al. who showed strong dependence of $\kappa$ on the SiNW roughness and phonon spectrum, while the 
dependency on diameter was not as strong [81]. Roughened SiNWs exhibit thermal conductivities below the Casimir limit, and scattering models must consider the full phonon spectrum and scattering behavior at each phonon frequency. Fractal geometry, a way to describe a repeating pattern at every length scale, would be one suitable way to treat such scattering, as calculated by Lü for copper and tungsten damascene structures [87]. Overall, comparing $\kappa$ vs diameter for SiNWs studied by different authors [29-31,36,48-50,77-79,81,88] (Fig. 5), $\kappa$ follows the Casimir limit for VLS grown SiNWs with smooth surfaces and circular cross-sections, while SiNWs with roughened surfaces due to electrochemical etching or chemical treatment, SiNWs with ion implantation, as well as e-beam defined SiNWs which have rectangular cross-section, follow a trend below the Casimir limit.

Apart from roughness considerations in terms of geometry, boundary scattering behavior can also highly depend on the surface treatment [88]. Glynn et al. [89] studied roughness of SiNWs using transmission electron microscopy, showing that the roughness features themselves may be intrinsically different from the rest of the SiNW, thereby introducing new scattering mechanisms. Finally, the cross-sectional shape of the nanowires and the crystal orientations of the surfaces [90] may have a role in boundary scattering which needs to be further explored.

\section{CONCLUSIONS}

In conclusion, we demonstrated the fabrication of suspended SiNWs below $20 \mathrm{~nm}$ width using EUV-IL and a top-down approach giving large and well-ordered arrays of SiNWs. Significant process-induced elastic strain was shown for SiNWs that were etched first and then clamped down by thermally evaporated metal, possibly due to thermal expansion of the sample during the deposition process. This has important implications in strain engineering since strain causes carrier mobility enhancement for certain crystal orientations in silicon. The thermal conductivity of SiNWs was measured using the Stokes/anti-Stokes intensity ratios which matched well with the independent measurement of applying the bulk silicon temperature dependency of the Stokes peak shift. This shows that intrinsic scattering is unmodified from the bulk. Strain also did not influence thermal conductivity. Thermal conductivities in the range of 0.04 to $1.5 \mathrm{~W} / \mathrm{m} \mathrm{K}$ were found, significantly lower than the bulk thermal conductivity and agreeing well with trends shown by other investigators. However, the thermal conductivities are much lower than expected using a diffuse boundary scattering model. It was shown by others and in this paper, that not only does LER play a role in further increasing the rate of boundary scattering, but also that longer range phonons can be scattered by constrictions at the ends of the nanowires. Such low thermal conductivities in SiNWs, combined with high conductivity and the Seebeck coefficient would give a very efficient thermoelectric material and could be used for thermal energy harvesting.

\section{ACKNOWLEDGMENTS}

D. Fan would like to thank T. Zabel for helpful discussions and M. Vockenhuber for assistance with the IL. Part of the work for this paper was performed at the Swiss Light Source, Paul Scherrer Institut. Funding was kindly provided by the Schweizerischer Nationalfonds zur Förderung der Wissenschaftlichen Forschung (SNF; Grant No. 200021_143969).
[1] S. Misra, L. Yu, W. Chen, M. Foldyna, and P. R. i Cabarrocas, A review on plasma-assisted VLS synthesis of silicon nanowires and radial junction solar cells, J. Phys. Appl. Phys. 47, 393001 (2014).

[2] B. Tian and C. M. Lieber, Design, synthesis, and characterization of novel nanowire structures for photovoltaics and intracellular probes, Pure Appl. Chem. 83, 2153 (2011).

[3] X. Zhang, C. W. Pinion, J. D. Christesen, C. J. Flynn, T. A. Celano, and J. F. Cahoon, Horizontal silicon nanowires with radial p-n junctions: A platform for unconventional solar cells, J. Phys. Chem. Lett. 4, 2002 (2013).

[4] F. Priolo, T. Gregorkiewicz, M. Galli, and T. F. Krauss, Silicon nanostructures for photonics and photovoltaics, Nat. Nanotechnol. 9, 19 (2014).

[5] T. Song, S.-T. Lee, and B. Sun, Silicon nanowires for photovoltaic applications: The progress and challenge, Nano Energy 1, 654 (2012).

[6] K.-Q. Peng, X. Wang, L. Li, Y. Hu, and S.-T. Lee, Silicon nanowires for advanced energy conversion and storage, Nano Today 8, 75 (2013).

[7] Y. Wang, T. Wang, P. Da, M. Xu, H. Wu, and G. Zheng, Silicon nanowires for biosensing, energy storage, and conversion, Adv. Mater. 25, 5177 (2013).
[8] Y. Wang, T. Li, and H. Yang, Nanofabrication, effects and sensors based on micro-electro-mechanical systems technology, Philos. Trans. R. Soc. Math. Phys. Eng. Sci. 371, 20120315 (2013).

[9] Q. Yu, H. Liu, and H. Chen, Vertical SiNWAs for biomedical and biotechnology applications, J. Mater. Chem. B 2, 7849 (2014).

[10] M. O. Noor and U. J. Krull, Silicon nanowires as field-effect transducers for biosensor development: A review, Anal. Chim. Acta 825, 1 (2014).

[11] A. Cao, E. Sudhölter, and L. de Smet, Silicon nanowire-based devices for gas-phase sensing, Sensors 14, 245 (2013).

[12] P. Namdari, H. Daraee, and A. Eatemadi, Recent advances in silicon nanowire biosensors: Synthesis methods, properties, and applications, Nanoscale Res. Lett. 11, 406 (2016).

[13] N. Lu, A. Gao, H. Zhou, Y. Wang, X. Yang, and Y. Wang, Progress in silicon nanowire-based field-effect transistor biosensors for label-free detection of DNA, Chin. J. Chem. 34, 308 (2016).

[14] R. M. Penner, Chemical sensing with nanowires, Annu. Rev. Anal. Chem. 5, 461 (2012).

[15] D. O. Demchenko, P. D. Heinz, and B. Lee, Determining factors of thermoelectric properties of semiconductor nanowires, Nanoscale Res. Lett. 6, 502 (2011). 
[16] A. Majumdar, Materials science: enhanced: Thermoelectricity in semiconductor nanostructures, Science 303, 777 (2004).

[17] A. M. Marconnet, M. Asheghi, and K. E. Goodson, From the Casimir limit to phononic crystals: 20 years of phonon transport studies using silicon-on-insulator technology, J. Heat Transf. 135, 61601 (2013).

[18] A. Martinez, M. Aldegunde, N. Seoane, A. R. Brown, J. R. Barker, and A. Asenov, Quantum-transport study on the impact of channel length and cross sections on variability induced by random discrete dopants in narrow gate-all-around silicon nanowire transistors, IEEE Trans. Electron Devices 58, 2209 (2011).

[19] I. Ferain, C. A. Colinge, and J.-P. Colinge, Multigate transistors as the future of classical metal-oxide-semiconductor field-effect transistors, Nature 479, 310 (2011).

[20] K. J. Kuhn, Considerations for ultimate CMOS scaling, IEEE Trans. Electron Devices 59, 1813 (2012).

[21] O. Hayden, R. Agarwal, and W. Lu, Semiconductor nanowire devices, Nano Today 3, 12 (2008).

[22] A. Fasoli and W. I. Milne, Overview and status of bottom-up silicon nanowire electronics, Mater. Sci. Semicond. Process. 15, 601 (2012)

[23] M. Hasan, M. F. Huq, and Z. H. Mahmood, A review on electronic and optical properties of silicon nanowire and its different growth techniques, SpringerPlus 2, 151 (2013).

[24] M. Amato and R. Rurali, Surface physics of semiconducting nanowires, Prog. Surf. Sci. 91, 1 (2016).

[25] N. S. Mohammad, Understanding quantum confinement in nanowires: Basics, applications and possible laws, J. Phys. Condens. Matter 26, 423202 (2014).

[26] M. Y. Bashouti, K. Sardashti, S. W. Schmitt, M. Pietsch, J. Ristein, H. Haick, and S. H. Christiansen, Oxide-free hybrid silicon nanowires: from fundamentals to applied nanotechnology, Prog. Surf. Sci. 88, 39 (2013).

[27] R. Geiger, T. Zabel, and H. Sigg, Group IV direct band gap photonics: Methods, challenges, and opportunities, Front. Mater. 2 (2015).

[28] L. D. Hicks and M. S. Dresselhaus, Thermoelectric figure of merit of a one-dimensional conductor, Phys. Rev. B 47, 16631 (1993).

[29] A. I. Boukai, Y. Bunimovich, J. Tahir-Kheli, J.-K. Yu, W. A. Goddard III, and J. R. Heath, Silicon nanowires as efficient thermoelectric materials, Nature 451, 168 (2008).

[30] A. I. Hochbaum, R. Chen, R. D. Delgado, W. Liang, E. C. Gartnett, M. Najarian, A. Majumdar, and P. Yang, Enhanced thermoelectric performance of rough silicon nanowires, Nature 451, 163 (2008).

[31] S. P. Rodichkina, L. A. Osminkina, M. Isaiev, A. V. Pavlikov, A. V. Zoteev, V. A. Georgobiani, K. A. Gonchar, A. N. Vasiliev, and V. Yu. Timoshenko, Raman diagnostics of photoinduced heating of silicon nanowires prepared by metal-assisted chemical etching, Appl. Phys. B 121, 337 (2015).

[32] H. Scheel, S. Reich, A. C. Ferrari, M. Cantoro, A. Colli, and C. Thomsen, Raman scattering on silicon nanowires: The thermal conductivity of the environment determines the optical phonon frequency, Appl. Phys. Lett. 88, 233114 (2006).

[33] S. Khachadorian, H. Scheel, M. Cantoro, A. Colli, A. C. Ferrari, and $\mathrm{C}$. Thomsen, The morphology of silicon nanowire samples: a Raman study: morphology of silicon nanowire samples, Phys. Status Solidi B 246, 2809 (2009).
[34] J. Anaya, A. Torres, V. Hortelano, J. Jiménez, A. C. Prieto, A. Rodríguez, T. Rodríguez, R. Rogel, and L. Pichon, Raman spectrum of Si nanowires: Temperature and phonon confinement effects, Appl. Phys. A 114, 1321 (2014).

[35] V. Poborchii, T. Tada, Y. Morita, and T. Kanayama, Ultraviolet Raman spectra of single uncoated and $\mathrm{SiO} 2$-coated silicon-oninsulator nanowires: Phonon boundary scattering, wave-vector relaxation and stress, Appl. Phys. Lett. 103, 153104 (2013).

[36] K. F. Murphy, B. Piccione, M. B. Zanjani, J. R. Lukes, and D. S. Gianola, Strain- and defect-mediated thermal conductivity in silicon nanowires, Nano Lett. 14, 3785 (2014).

[37] M. Khorasaninejad, M. M. Adachi, J. Walia, K. S. Karim, and S. S. Saini, Raman spectroscopy of core/shell silicon nanowires grown on different substrates, Phys. Status Solidi A 210, 373 (2013).

[38] R.-p. Wang, G.-w. Zhou, Y.-1. Liu, S.-h. Pan, H.-z. Zhang, D.-p. $\mathrm{Yu}$, and Z. Zhang, Raman spectral study of silicon nanowires: High-order scattering and phonon confinement effects, Phys. Rev. B 61, 16827 (2000).

[39] S. Piscanec, M. Cantoro, A. C. Ferrari, J. A. Zapien, Y. Lifshitz, S. T. Lee, S. Hofmann, and J. Robertson, Raman spectroscopy of silicon nanowires, Phys. Rev. B 68, 241312(R) (2003).

[40] K. W. Adu, M. D. Williams, M. Reber, R. Jayasingha, H. R. Gutierrez, and G. U. Sumanasekera, Probing phonons in nonpolar semiconducting nanowires with Raman spectroscopy, J. Nanotechnol. 2012, 264198 (2012).

[41] J. S. Heron, T. Fournier, N. Mingo, and O. Bourgeois, Mesoscopic size effects on the thermal conductance of silicon nanowire, Nano Lett. 9, 1861 (2009).

[42] A. Nikolenko, V. Strelchuk, A. Klimovskaya, P. Lytvyn, M. Valakh, Y. Pedchenko, A. Voroschenko, and D. Hourlier, Scanning confocal Raman spectroscopy of silicon phase distribution in individual Si nanowires, Phys. Status Solidi C 8, 1012 (2011).

[43] A. T. Chu, T. T. N. Thi, T. T. Tran, B. N. Vu, T. T. Pham, V. T. Pham, T. H. Pham, and H. D. Pham, Raman photoluminescence spectra of silicon nanowires synthesized by a vapor phase transport method, Adv. Nat. Sci. Nanosci. Nanotechnol. 2, 35004 (2011).

[44] G. S. Doerk, C. Carraro, and R. Maboudian, Temperature dependence of Raman spectra for individual silicon nanowires, Phys. Rev. B 80, 073306 (2009).

[45] L. A. Golovan, K. A. Gonchar, L. A. Osminkina, V. Y. Timoshenko, G. I. Petrov, and V. V. Yakovlev, Coherent antiStokes Raman scattering in silicon nanowire ensembles, Laser Phys. Lett. 9, 145 (2012).

[46] V. Y. Timoshenko, K. A. Gonchar, L. A. Golovan, A. I. Efimova, V. A. Sivakov, A. Dellith, and S. A. Christiansen, Photoluminescence and Raman scattering in arrays of silicon nanowires, J. Nanoelectron. Optoelectron. 6, 519 (2011).

[47] K. Oda, Y. Nanai, T. Sato, S. Kimura, and T. Okuno, Correlation between photoluminescence and structure in silicon nanowires fabricated by metal-assisted etching: correlation between photoluminescence and structure in silicon nanowires, Phys. Status Solidi A 211, 848 (2014).

[48] D. Li, Y. Wu, P. Kim, L. Shi, P. Yang, and A. Majumdar, Thermal conductivity of individual silicon nanowires, Appl. Phys. Lett. 83, 2934 (2003).

[49] S. Toda, T. Oishi, T. Yoshioka, and T. Okuno, Optical properties of silicon nanowires fabricated by electroless silver deposition, Jpn. J. Appl. Phys. 49, 95002 (2010). 
[50] Z. Wang, X. Chen, Q. Yan, and J. Zhang, Raman study on thermal conductivity of oxide-coated silicon nanowires, Europhys. Lett. 100, 14002 (2012).

[51] H. Richter, Z. P. Wang, and L. Ley, The one phonon Raman spectrum in microcrystalline silicon, Solid State Commun. 39, 625 (1981).

[52] I. H. Campbell and P. M. Fauchet, The effects of microcrystal size and shape on the one phonon Raman spectra of crystalline semiconductors, Solid State Commun. 58, 739 (1986).

[53] J. Zi, H. Büscher, C. Falter, W. Ludwig, K. Zhang, and X. Xie, Raman shifts in Si nanocrystals, Appl. Phys. Lett. 69, 200 (1996).

[54] A. Tarun, N. Hayazawa, M. V. Balois, S. Kawata, M. Reiche, and $\mathrm{O}$. Moutanabbir, Stress redistribution in individual ultrathin strained silicon nanowires: A high-resolution polarized Raman study, New J. Phys. 15, 53042 (2013).

[55] M. V. Balois, N. Hayazawa, A. Tarun, S. Kawata, M. Reiche, and O. Moutanabbir, Direct optical mapping of anisotropic stresses in nanowires using transverse optical phonon splitting, Nano Lett. 14, 3793 (2014).

[56] I. D. Wolf, Micro-Raman spectroscopy to study local mechanical stress in silicon integrated circuits, Semicond. Sci. Technol. 11, 139 (1996).

[57] J. Anaya, A. Torres, A. Martín-Martín, J. Souto, J. Jiménez, A. Rodríguez, and T. Rodríguez, Study of the temperature distribution in $\mathrm{Si}$ nanowires under microscopic laser beam excitation, Appl. Phys. A 113, 167 (2013).

[58] C. Wagner and N. Harned, EUV lithography: Lithography gets extreme, Nat. Photonics 4, 24 (2010).

[59] G. Tallents, E. Wagenaars, and G. Pert, Optical lithography: Lithography at EUV wavelengths, Nat. Photonics 4, 809 (2010).

[60] N. Mojarad, J. Gobrecht, and Y. Ekinci, Interference lithography at EUV and soft $\mathrm{x}$-ray wavelengths: Principles, methods, and applications, Microelectron. Eng. 143, 55 (2015).

[61] E. Buitrago, R. Fallica, D. Fan, W. Karim, M. Vockenhuber, J. van Bokhoven, and Y. Ekinci, Extreme-UV interference lithography at the Paul Scherrer Institute, SPIE Newsroom 2016.

[62] D. Fan and Y. Ekinci, Photolithography reaches $6 \mathrm{~nm}$ half-pitch using extreme ultraviolet light, J. MicroNanolithography MEMS MOEMS 15, 33505 (2016).

[63] See Supplemental Material at http://link.aps.org/supplemental/ 10.1103/PhysRevB.96.115307 for thermal modeling and simulation, laser power absorption calculations, and supporting Raman measurements.

[64] M. G. Moharam and T. K. Gaylord, Rigorous coupled-wave analysis of planar-grating diffraction, J. Opt. Soc. Am. 71, 811 (1981).

[65] M. A. Green and M. J. Keevers, Optical properties of intrinsic silicon at 300 K, Prog. Photovolt. Res. Appl. 3, 189 (1995).

[66] I. H. Malitson, Interspecimen comparison of the refractive index of fused silica, J. Opt. Soc. Am. 55, 1205 (1965).

[67] A. D. Rakić, A. B. Djurišić, J. M. Elazar, and M. L. Majewski, Optical properties of metallic films for vertical-cavity optoelectronic devices, Appl. Opt. 37, 5271 (1998).

[68] H. H. Burke and I. P. Herman, Temperature dependence of Raman scattering in $\mathrm{Ge}_{1-x} \mathrm{Si}_{x}$ alloys, Phys. Rev. B 48, 15016 (1993).
[69] V. V. Brazhkin, S. G. Lyapin, I. A. Trojan, R. N. Voloshin, A. G. Lyapin, and N. N. Mel'nik, Anharmonicity of short-wavelength acoustic phonons in silicon at high temperatures, J. Exp. Theor. Phys. Lett. 72, 195 (2000).

[70] B. A. Weinstein and G. J. Piermarini, Raman scattering and phonon dispersion in $\mathrm{Si}$ and $\mathrm{GaP}$ at very high pressure, Phys. Rev. B 12, 1172 (1975)

[71] M. J. Süess, R. A. Minamisawa, R. Geiger, K. K. Bourdelle, H. Sigg, and R. Spolenak, Power-dependent Raman analysis of highly strained Si nanobridges, Nano Lett. 14, 1249 (2014).

[72] R. A. Minamisawa, M. J. Süess, R. Spolenak, J. Faist, C. David, J. Gobrecht, K. K. Bourdelle, and H. Sigg, Top-down fabricated silicon nanowires under tensile elastic strain up to $4.5 \%$, Nat. Commun. 3, 1096 (2012).

[73] M. Chu, Y. Sun, U. Aghoram, and S. E. Thompson, Strain: a solution for higher carrier mobility in nanoscale MOSFETs, Annu. Rev. Mater. Res. 39, 203 (2009).

[74] M. Bruel, B. Aspar, and A.-J. Auberton-Hervé, Smart-cut: A new silicon on insulator material technology based on hydrogen implantation and wafer bonding, Jpn. J. Appl. Phys. 36, 1636 (1997).

[75] Y. A. Çengel and A. J. Ghajar, Heat and Mass Transfer: Fundamentals and Applications, 5th ed. (McGraw-Hill Education, New York, 2015).

[76] Distribution Page of RODIS, Photonics Research Group, Ghent University, www.photonics.intec.ugent.be/research/facilities/ design/rodis/default.htm, (2003).

[77] J.-K. Yu, S. Mitrovic, D. Tham, J. Varghese, and J. R. Heath, Reduction of thermal conductivity in phononic nanomesh structures, Nat. Nanotechnol. 5, 718 (2010).

[78] K. Hippalgaonkar, B. Huang, R. Chen, K. Sawyer, P. Ercius, and A. Majumdar, Fabrication of microdevices with integrated nanowires for investigating low-dimensional phonon transport, Nano Lett. 10, 4341 (2010).

[79] J. P. Feser, J. S. Sadhu, B. P. Azeredo, K. H. Hsu, J. Ma, J. Kim, M. Seong, N. X. Fang, X. Li, P. M. Ferreira, S. Sinha, and D. G. Cahill, Thermal conductivity of silicon nanowire arrays with controlled roughness, J. Appl. Phys. 112, 114306 (2012).

[80] J.-S. Heron, C. Bera, T. Fournier, N. Mingo, and O. Bourgeois, Blocking phonons via nanoscale geometrical design, Phys. Rev. B 82, 155458 (2010).

[81] J. Lim, K. Hippalgaonkar, S. C. Andrews, A. Majumdar, and P. Yang, Quantifying surface roughness effects on phonon transport in silicon nanowires, Nano Lett. 12, 2475 (2012).

[82] P. Martin, Z. Aksamija, E. Pop, and U. Ravaioli, Impact of Phonon-Surface Roughness Scattering on Thermal Conductivity of Thin Si Nanowires, Phys. Rev. Lett. 102, 125503 (2009).

[83] J. Zou and A. Balandin, Phonon heat conduction in a semiconductor nanowire, J. Appl. Phys. 89, 2932 (2001).

[84] A. L. Moore, S. K. Saha, R. S. Prasher, and L. Shi, Phonon backscattering and thermal conductivity suppression in sawtooth nanowires, Appl. Phys. Lett. 93, 83112 (2008).

[85] Z. Wang, J. E. Alaniz, W. Jang, J. E. Garay, and C. Dames, Thermal conductivity of nanocrystalline silicon: Importance of grain size and frequency-dependent mean free paths, Nano Lett. 11, 2206 (2011).

[86] C. Hua and A. J. Minnich, Importance of frequency-dependent grain boundary scattering in nanocrystalline silicon and silicon-germanium thermoelectrics, Semicond. Sci. Technol. 29, 124004 (2014). 
[87] X. Lü, Thermal conductivity modeling of copper and tungsten damascene structures, J. Appl. Phys. 105, 94301 (2009).

[88] Y. Pan, Y. Tao, G. Qin, Y. Fedoryshyn, S. N. Raja, M. Hu, C. L. Degen, and D. Poulikakos, Surface chemical tuning of phonon and electron transport in free-standing silicon nanowire arrays, Nano Lett. 16, 6364 (2016).
[89] C. Glynn, K.-M. Jones, V. Mogili, W. McSweeney, and C. O'Dwyer, The nature of silicon nanowire roughness and thermal conductivity suppression by phonon scattering mechanisms, ECS J. Solid State Sci. Technol. 6, N3029 (2017).

[90] Y. Zhou, Y. Chen, and M. Hu, Strong surface orientation dependent thermal transport in Si nanowires, Sci. Rep. 6, 24903 (2016). 